\section{Catastrophe theory}

Sir,-It is not my purpose here to discuss in detail the criticisms of catastrophe theory contained in the review article by $H$. Sussman and R. Zahler (27 October, page 759). I would like to refer the interested reader, however, to a forthcoming article of mine entitled 'Mathématique et théorisation scientifique' to appear in Scientia. I would also like to point out a misquotation by the authors. The classification theorem for the "Cusp catastrophe", erroneously quoted as "Thom's theorem", is in this specific case due to $\mathbf{H}$. Whitney (Mapping of the plane into the plane, Ann. of Math. 2, 62, pp. 374-410 (1955)).

\section{RENE THOM}

Institut des Hautes Etudes Scientifiques, France

SIR,-I would like to make two criticisms concerning Zahler and Sussman's recent review article on the applications of catastrophe theory (27 October, page 759). The first is that an editorial policy which sanctions the publication of polemical and emotive articles has no place in a scientific journal. In my view, the tone of Zahler and Sussman's comments goes beyond the lively discussions which we all welcome. Despite its many successes, there have been incorrect examples and applications of Thom's theory and extravagant claims for it. These should be assessed carefully and any errors rebutted scientifically. Zahler and Sussman's excessive and misleading criticisms do not help in the proper evaluation of the usefulness of Thom's theory and can only encourage a polarisation of opinion.

My second criticism is of the poor standard of argument and exposition in the paper and some comments on the first two pages follow. First Zahler and Sussman state that the record of legitimate uses of Thom's theory in physics and engineering is poor. But the theory has been applied very successfully in engineering ${ }^{2,3}$ and optics ${ }^{4}$.

Next, Zahler and Sussman appear to have missed the real weakness in the claim by Kozak and Benham ${ }^{5}$ and as a result much of their criticism is irrelevant. Thom's theory applies to processes governed by a potential function and with at most five controls, and which no matter how slowly the controls cross a threshold, pass from one equilibrium state to another through transitional states which are not in equilibrium. This passage between equilibrium states is continuous and often, though by no means always, very fast, particularly in mechanical cases, but never instantaneous. The discontinuities or 'jumps' arise if only equilibrium states are measured. Thom's theory does not apply if, when the controls are varied slowly enough, the process remains in equilibrium throughout the transition. Consequently it is quite mistaken to suggest that the theory offers the cusp catastrophe as 'an inevitable, universal paradigm' for any system which exhibits sudden changes associated with two control parameters. Indeed because the process of collagen denaturation described by Kozak and Benham is of this kind ${ }^{6,7}$, Thom's theory does not apply and one cannot expect collagen denaturation to conform to the cusp or any other catastrophe. The arguments based on the van't Hoff equation which Zahler and Sussman make in the section aptly headed 'Confusion about continuity' are quite irrelevant since the equation holds only under equilibrium conditions.

They go on to assert that 'most biological situations which catastrophe theory tries to model' [sic] are 'inherently continuous'. This is certainly true in the sense that the transition from one state to another, no matter how sudden, is still continuous at least above the quantum level, even if the process is not in equilibrium during the transition. It is not true if by inherently continuous Zahler and Sussman mean (as I think the context indicates that they do) that the transition states of such processes are equilibrium states. Genetic assimilation and quantum evolution are two important biological processes which involve transition states which are not in equilibrium. Moreover the concept of fitness and the widely used selection landscape of Simpson and Wright $^{8}$ imply that evolution commonly proceeds from one maximally fit form to another through intermediate forms which are not maximally fit, that is not in equilibrium with their environment.

In fact, contrary to Zahler and Sussman's assertions on page 762 about my work, the notions of fitness and selection landscape permit Thom's theory to be incorporated naturally into the analysis of the adaptive response of populations subject to natural selection in slowly varying environments. This has been done for quantum evolution ${ }^{9}$, genetic assimilation $^{10}$ and allopatric speciation ${ }^{11}$. Two of these papers contain quantitative predictions for the response of the phenotype, although it is true that the imperfection of the fossil record and the variability in populations present great practical difficulties. However, these are problems which are common in applying mathematics to biology and are not peculiar to catastrophe theory.

Similarly in his work, Zeeman assumes a gradient system (a common enough practice in biology) so that Thom's theory can be applied. Moreover he makes many quantitative predictions which depend on the properties of the cusp catastrophe. There are a number made in 'Primary and secondary waves in developmental biology'12, cited by Zahler and Sussman, particularly in $\$ 9$ and $\$ 15$. It is a limitation of the theory that some of these predictions can only be made to first order, as Zeeman makes clear in the second sentence of $\$ 9$. Incidentally Zahler and Sussman's remarks about Zeno's paradox suggest that they have not appreciated this nor that at its vertex the cusp is flat. These predictions, in contrast to Zahler and Sussman's assertions, are not contained in the data, not independent of the theory and not just wrong. They need, as Zeeman suggests explicitly, to be tested experimentally and Zahler and Sussman's description of these predictions as 'purely unverified hopes' is a gross misrepresentation of what Zeeman has said, as reference to the paper just cited will show.

Zahler and Sussman do not make it clear that in spite of the underlying continuity, many important biological phenomena, such as somites, boundaries between different tissues, etc., are discrete and discontinuous. This provided a considerable stimulus to Thom's thinking and it is no accident that his theory offers a possible way of handling such phenomena mathematically. The most profitable procedure is to test the predictions which follow from applications of the theory and to see whether its geometrical framework gives useful insights.

A general comment is that the accomplishments or otherwise of the theory can be better assessed if a clear distinction is made between an example and an application, and between an explanation and a description.

University of York, UK

Maurice Dodson

1. Zahler, R. S. \& Sussman, H. J. Nature 269, 759 (1977)

2. Thompson, J. M. T. Z.A.M.P. 26, 581 (1975).

. Thompson, J. M. T. Nature 254, 392 (1975).

4. Berry, M. V. \& Nye, J. F. Nature 267, 34 (1977). Kozak, J. J. \& Benham, C.

6. von Hippel, P. H. \& Kwok-Ying Wong Biochem. 1, 664 (1962).

von Hippel, $\mathrm{P}$.

2, 1387 (1963) Simpson, G. G. The Major Features Columbia University Press, 1953

9. Dodson, M. M. Evolut. Theory 1, 107 (1975).

11. Dodson, M. M. \& Hallam, A. Amer. Nat. 111 415 (1977).

12. Zeeman, E. C. in Lectures on Mathematics in the Life Sciences 7, 69 (1974).

\title{
Colour genes
}

SIR,-Our ignorance of the genetic basis of skin colour is not as profound as implied in Dr Bowne's letter (13 October, page 556). Nor do we have to assume the existence of unknown genes closely linked to colour genes in order to explain other traits that might be associated with skin colour, for there is evidence suggesting that the vast majority of pigmentation loci are probably pleiotropic (Deol, M. S. Ann. Hum. Genet., 38, 501 (1975)).

Dr Bowne's views on intelligence are, of course, unexceptionable. Yours faithfully, M. S. DEOL

University College, London 